Thorax 1984;39:558-559

\title{
Innominate artery fistula complicating cervical gastrostomy
}

\section{KM PAGLIERO, TH PETERS}

\author{
From the Royal Devon and Exeter Hospital, Exeter
}

Cervical gastrostomy has recently been advocated in management of patients after subtotal oesophagectomy.' We report a case where this manoeuvre was complicated by the development of an innominate arteriogastrocutaneous fistula.

\section{Case report}

A 69 year old woman was found to have a squamous cell carcinoma of the oesophagus, assessed as operable, $28 \mathrm{~cm}$ from the incisor teeth. It was excised by a synchronous approach via midline abdominal, right anterolateral thoracic, and right oblique cervical incisions, with oesophagogastric anastomosis just distal to the cricopharyngeal sphincter. Pyloroplasty was performed. A cervical gastrostomy was constructed with a Salem sump tube, size 18 (Argyle Division, for Sherwood Medical Industries, Belgium), in the manner described by Barros, ${ }^{1}$ the tube being brought out lateral to the cervical incision. A cervical wound drain was inserted medial to the incision and withdrawn after four days as there was no evidence of anastomotic leakage. An abdominal jejunostomy was provided for feeding. Bowel motility returned at the normal time but severe cardiorespiratory distress on the first postoperative day demanded continued mechanical ventilation via an endotracheal tube. There was little subsequent improvement and the gastrostomy tube was not removed. After 10 days the feeding jejunostomy tube became blocked and the gastrostomy tube was used as a feeding route.

Fourteen days after operation acute shock developed, accompanied by haematemesis, melaena, and pulsatile bleeding from around the gastrostomy tube. Immediate operation was performed via the cervical incision and a communication was found between the tract and the innominate artery. After control of the head vessels had been gained the innominate artery was excised and its stump and the proximal end of the right subclavian and external carotid artery were transfixed, ligated, and allowed to recoil away from the track site. The gastrostomy tube was removed and the stomach closed. Bleeding was controlled and subsequent neurological examination showed nothing abnormal, indicating that the patient had an intact circle of Willis supplied from the other side.

Address for reprint requests: Mr KM Pagliero, Royal Devon and Exeter Hospital, Exeter EX2 5DW.

Accepted 2 April 1984
Despite this the respiratory state continued to deteriorate for another $\mathbf{2 3}$ days, and $\mathbf{3 8}$ days after the initial operation there was more bleeding from the gastrostomy site. A further operation was not performed as the patient's respiratory state was considered irreversible and she died on the 39 th day.

\section{Discuscion}

Like Barros,' we are persuaded by Argov $^{2}$ of the importance of minimising aspiration pneumonitis by keeping the stomach empty after oesophagectomy. This may prevent anastomotic leakage by reducing the tension on the anastomosis that might be caused by a partially full stomach. We agree with Belsey (quoted by Barrosi) that a nasogastric tube renders the upper oesophageal sphincter incompetent and also consider that it produces an inflammatory exudation that can cause considerable discomfort to the patient and may contribute to the aspiration problems. For these reasons many prefer to rely on a pyloric drainage procedure alone and in our experience most patients produce only small amounts of aspirate. There continue, however, to be a few who develop a prolonged ileus, for whom tube drainage is essential. This raises the question whether to use tube drainage in all cases or whether to use it only as required and risk damage to the anastomosis during insertion. Barros's technique' circumvents this dilemma and we readily adopted it until the serious complication we have described.

Before we condemn the procedure it is important to ensure that the method was accurately reproduced. Our technique differed from that of Barros in three ways. Firstly, our gastrostomy was brought out lateral to the wound rather than medial. We do not consider this important. The length of track would be similar and by either route the head vessels would be similarly adjacent. The presence of a separate wound drain we regard as irrelevant, as it was well away from the gastrostomy tube and was removed early, long before the complication, and at no time was it the site of discharge or bleeding. Secondly, we preferred to use a sump drain for optimum gastric emptying and therefore used $a$ hard plastic tube rather than the soft rubber catheter used by Barros. While the material of our tube is probably less irritant than rubber, conceivably the rigidity may have contributed to the complication. Nevertheless, in 11 other cases in which we have used the technique we have had no problems. Thirdly, our patient differs from those of Barros and our other 11 patients in 
the length of time the gastrostomy tube was left in place. We now think that the period of more than two weeks was inappropriate. Normally there would be no need to retain the tube after satisfactory gastric emptying had returned and our present view is that these tubes should be removed as soon as possible after time has been allowed for a track to be established (a minimum of 5 days).

The cause of this patient's complication is presumably a combination of the physical trauma of the tube itself and the nature of the fluid exuding around the tube. This fluid is irritant, containing pepsin at low $\mathrm{pH}$, and it may also be infected, though it did not appear to be in our case as there was no discharge from the stoma before the haemorrhage and no pus at exploration. Barros, however, describes a case of cervical abscess, albeit successfully managed, in one of his six cases. Delayed removal of the tube would seem to increase the likelihood that these factors will become important. One such complication in 12 cases is an unacceptable incidence. It is, therefore, important that it should be recorded. The benefits of comfort for the patient and trouble free respiratory care in the 11 cases in which we use this technique successfully make us hope for further reports suggesting this complication is exceptional. Meanwhile we must view the technique with extreme caution.

\section{References}

' Barros JL. Cervical gastrostomy in the surgical treatment of oesophageal cancer. Thorax 1981;36:863-5.

${ }^{2}$ Argov S, Goldstein I, Barzilla O. Is routine use of the nasogastric tube justified in upper abdominal surgery? Am J Surg 1980;139:849-50. 